\title{
Fast Bistable Intensive Light Scattering in Helix-Free Ferroelectric Liquid Crystals
}

\author{
Alexander Andreev ${ }^{1}$, Tatiana Andreeva ${ }^{1}$, Igor Kompanets ${ }^{1,2, *}$, \\ Nikolay Zalyapin², Huan $\mathrm{Xu}^{3}$, Mike Pivnenko ${ }^{3}$, Daping $\mathrm{Chu}^{3}$ \\ ${ }^{1}$ P. N. Lebedev Physical Institute (LPI), 53 Leninsky pr., Moscow 119991, Russia, \\ ${ }^{2}$ National Research Nuclear University "MEPhI", 31 Kashirskoe shosse, Moscow 115409, Russia \\ ${ }^{3}$ University of Cambridge, CAPE, $9 \mathrm{JJ}$ Thomson Avenue, Cambridge, CB3 OFA, UK \\ *Corresponding author: kompan@sci.lebedev.ru
}

Received XX Month XXXX; revised XX Month, XXXX; accepted XX Month XXXX; posted XX Month XXXX (Doc. ID XXXXX); published XX Month XXXX

\begin{abstract}
A new type of ferroelectric liquid crystals (FLC) is considered, where reorientation of the director (main optical axes) is due to the movement of spatially localized waves with a stationary profile - solitons arising at the transition to the Maxwellian mechanism of energy dissipation. Under certain conditions, the appearance of such waves leads to the formation of a structure of transient domains, and as a consequence, to the scattering of light. The Maxwellian mechanism of energy dissipation allows to reduce the electric field strength, at which the maximum efficiency of light scattering is achieved, down to $2 \div 3 \mathrm{~V} / \mu \mathrm{m}$, and to increase the frequency of light modulation up to $3 \div 5 \mathrm{kHz}$. The intensive bistable light scattering in an electro optical cell filled with a specially designed helix-free FLC was studied, and a stable scattering state can be switched on and off for a few tens of microseconds and memorized for a few tens of seconds. (C) 2015 Optical Society of America
\end{abstract}

OCIS codes: (230.2090) Electro-optical devices; (290.0290) Scattering; (160.3710) Liquid crystals; (160.2260) Ferroelectrics.

http://dx.doi.org/

\section{INTRODUCTION}

Electrically controlled optical shutters and displays without using polarizer are very desirable in order to obtain high optical efficiency, especially in IR region. One of the approaches to achieve such devices is utilizing the phenomena of intensive and fast light scattering previously found in ferroelectric liquid crystals (FLCs) [1].

In our past work [1], we reported the electro-optical response of dynamic light scattering observed in a test cell filled with a helical FLC with spontaneous polarization $P_{s}>50$ $\mathrm{nC} / \mathrm{cm}^{2}$. The helix winding means that the azimuth angle of the director orientation varies continuously from 0 to $2 \pi$ along the direction orthogonal to smectic layers at a distance equal to the helix pitch. Smectic layers are considered as periodic ordering of mass centers of molecules in the direction of the director with a period of the order of FLC molecule's length.

The scattering phenomenon was characterized by rather fast speed (scattering ON and OFF times smaller than $150 \mu$ s under $E=5 \mathrm{~V} / \mu \mathrm{m}$ ), quite high light scattering efficiency (defined as the contrast ratio between cleared and scattered states, which exceeded 100:1), as well as bi-stable operation mode with memorizing any of optical states for a few milliseconds after an electric field turned off [1].
We introduce here a new type of light scattering materials of optimized electro optical performances. Scattering of light in them occurs at the boundaries of spontaneously ordered regions, which are formed in helix-free FLC due to arising the waves of a stationary profile - solitons.

The materials are specially formulated helix-free FLCs with spontaneous polarization $P_{s}<50 \mathrm{nC} / \mathrm{cm}^{2}$. The original helical structure was suppressed completely by introducing chiral impurities possessing opposite sign of optical activity. In such FLCs at definite conditions deformations of smectic layers arise, which result in the periodical changes of the director orientation along smectic layers in the absent of an electric field. Besides, the spatially localized waves of a stationary profile - solitons appear due to the transition to the Maxwellian mechanism of energy dissipation $[2,3]$.

An alternating electric field $\boldsymbol{E}$ is created by bipolar voltage applied to transparent electrodes on both substrates of an electro-optical FLC cell that is directed along smectic layers. $\boldsymbol{E}$ interacts with FLC spontaneous polarization and changes the director distribution in each layer. Development of this process in the electric field results in appearance of a soliton, which is the steady wave packet with a localized periodic wave. Motion of solitons in smectic layers reorients the director in all FLC volume. The reorientation of the director means the modulation of the phase delay between the ordinary and 
extraordinary rays or the modulation of light intensity if an electro-optical cell is placed between crossed polarizers. Transition to the soliton mode provides the frequency of light modulation up to $7 \mathrm{kHz}$ at the control voltage amplitude of 1.5 $\mathrm{V}$ and the thickness of electro-optical cells of about $1.7 \mu \mathrm{m}$. In this case, the electro-optical response time does not exceed 30 microseconds [4].

The appearance of the waves of stationary profile leads to the formation of the structure of transient domains (spontaneously ordered regions), and light scattering takes place at the boundaries of the transient domains. The soliton motion under an electric field causes the reorientation of the molecular director in the entire volume of FLC, and as a consequence, the transient domains disappear. If the plane of polarization of the incident light is along the direction of FLC director (along the main optical axis of FLC layer), then the light transmittance of an electrooptical cell becomes maximum.

The inversion of electric field sign (i. e. pulse polarity of the control voltage) induces the soliton wave's formation that leads to appearance of refraction index gradients along the smectic layers that is accompanied by intensive light scattering. In addition, the above-described process of scattering allows operating in the bistable mode, when switching of an electrooptical modulator from scattering to transparent state and back is carried out by changing the polarity of the control voltage pulse. Moreover both the optical states were found to remain for a few tens of seconds after the electric field is switched off or until the arrival of the pulse of reverse polarity.

In this work we studied the helix-free FLC with the following material parameters: spontaneous polarization $P_{S}=40$ $\mathrm{nC} / \mathrm{cm}^{2}$, rotational viscosity coefficient $\gamma_{\varphi}=0.7$ Poise, tilt angle of molecules in smectic layers $\Theta_{0}=23^{\circ}$ (at $20^{\circ} \mathrm{C}$ ), temperature range of the ferroelectric (chiral smectic $\mathrm{C}^{*}$ ) phase existence from $2{ }^{\circ} \mathrm{C}$ to $70{ }^{\circ} \mathrm{C}$.

\section{DEFORMATION OF SMECTIC LAYERS}

The existence of spatially-inhomogeneous layered structures in FLC volume is the reason for causing the deviation of director axes from their uniform distribution in FLC volume, and as a consequence the variation of a position of the main optical axes of the ellipsoid of refraction indexes along the direction those is orthogonal (in case of helix winding) or parallel (in case of helix suppression) to the smectic layers. In both cases the birefringence value of FLC depends on the frequency of an applied electric field $\boldsymbol{E}$.

For a helix-free FLC the physical reason resulting in the frequency dependence of its birefringence variation is the periodical deformation of smectic layers $[2,4]$. If FLC director orientation is homeotropic, i.e. the smectic layers are parallel to FLC cell substrates (in planar orientation the smectic layers are orthogonal to substrates), then the appearance of the deformation looks like the periodically alternating stripes with a period of $1.5 \div 7 \mu \mathrm{m}$, which depends on the molecular structure of FLC (Figure 1).

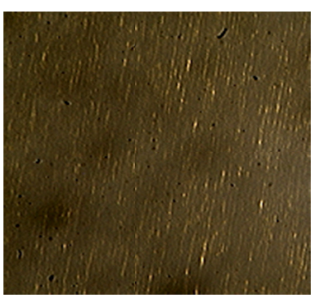

a)

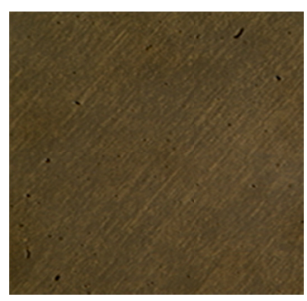

b)
Fig. 1. The periodic deformation of smectic layers of helix-free FLC in the absence of an electric field (a) and at field intensity of $0.05 \mathrm{~V} / \mu \mathrm{m}$ (b). Homeotropic orientations of the director, polarizers are crossed.

The existence of such deformations means that FLC molecules in smectic layers tilted initially by the angle $\Theta_{0}$ relative to the normal of the layer are declined additionally by an angle $\psi$ relative to the substrate's plane, i.e. relative to the axis $\mathbf{z}$ for the planar orientation (Figure 2).

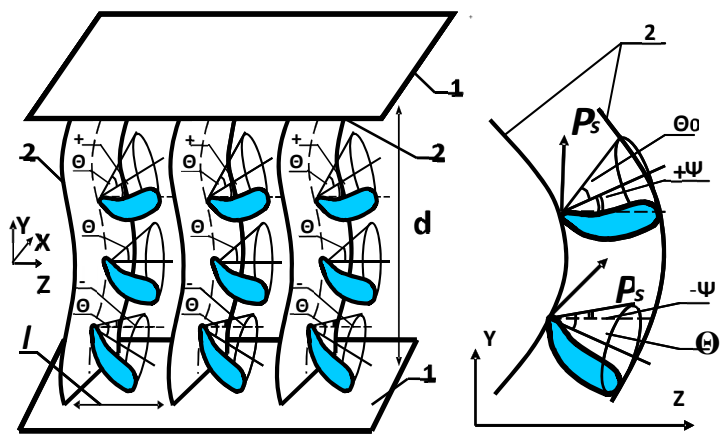

Fig. 2. Helix-free FLC with planar orientation of the director in an electro-optical cell. 1 - glass substrates with conductive covers, 2 - smectic layers, $\Theta_{0}$ - the angle of molecule's tilt in smectic layers, $\psi$ - the angle of tilt of a smectic layer, $\boldsymbol{P}_{s}$ - vector of the spontaneous polarization, $d$ - FLC cell thickness, $l$ smectic layer thickness.

Consequently the projection of FLC director onto the plane $\boldsymbol{x} \boldsymbol{y}$ is changed. The thickness of the bended smectic layer in the projection onto axis $\mathbf{z}$ is

Further we consider helix-free FLC cells only with planar orientation of the director.

\section{THE ENERGY OF SMECTIC LAYERS DEFORMATION}

For helix-free FLC depending on the sign of the electric field one of two homogeneous orientations of dipole molecules is realized. Two peaks of repolarization current $I P$ correspond to two values of the electric field of a different sign (Figure 3), at which re-polarization of the structure (reorientation of the vector $P_{S}$ of spontaneous polarization) takes place.

For FLC with periodic deformations of smectic layers at decreasing the electric field intensity from $+E_{\max }$ to zero (or from $-E_{\max }$ to zero) there is a part of the dependence $I_{P}(E)$ where a value of the current $I_{P}$ does not dependent practically on the field intensity (Figure 3a).

Accordingly, the dielectric susceptibility $\chi=d P / d E=I_{P} / S$ $(d E / d t)$, where $S$ - the area of cell electrodes, and does not also depend on the field intensity. This is the static value $\chi_{\text {st }}$ of the dielectric susceptibility associated with the elastic (reversible) deformation of the structure in an electric field. In the absence of periodic deformations of layers the static part of the dependence $I_{P}(E)$ is absent (Fig. $3 \mathrm{~b}$ ).

As in the case of the elastic deformation of the molecular structure with helical winding of the director $[5,6]$, the susceptibility $\chi_{\text {st }}$ corresponds to the linear response of the structure to the applied electric field and does not depend on the field strength:

$$
\chi_{s t}=\frac{1}{2} \frac{P_{S}^{2}}{\Theta_{0}{ }^{2} M}
$$




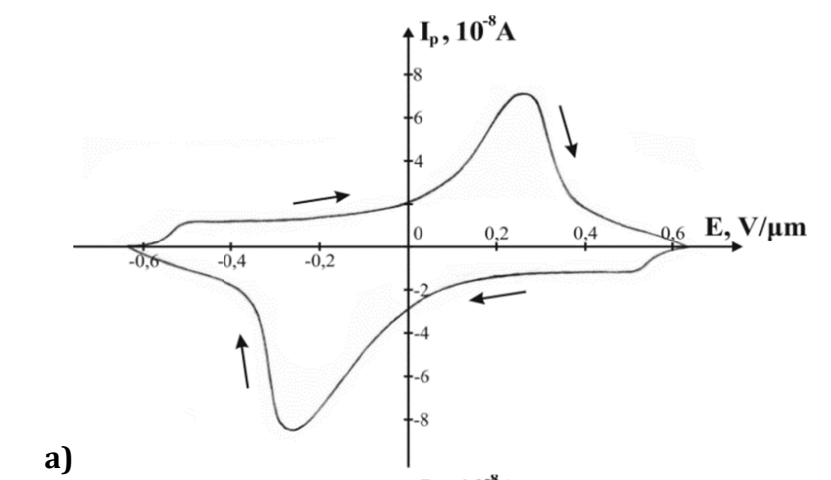

a)

\section{b)}

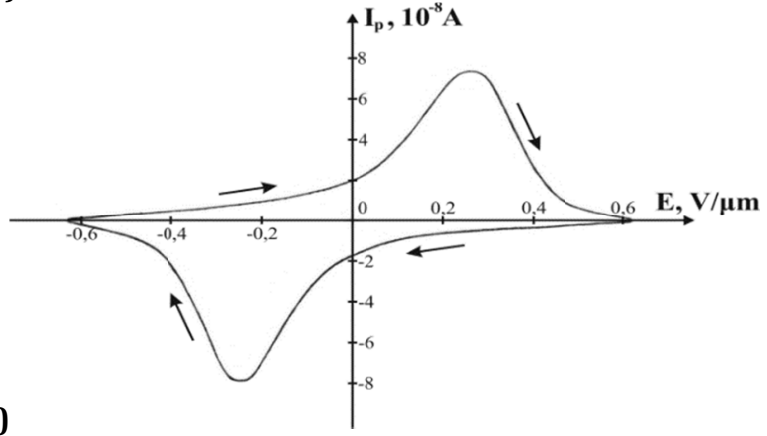

Fig. 3. Dependences of the repolarization current $I_{P}$ of helix-free FLC on the electric field intensity $E$ for structures with periodic deformations of smectic layers (a) and in the absence of layers deformation (b). The frequency of triangular shape bipolar voltage $f=5 \cdot 10^{-3} \mathrm{~Hz}$. The thickness of FLC cell $d=4 \mu \mathrm{m}$.

$$
\chi_{s t}=\frac{1}{2} \frac{P_{S}^{2}}{\Theta_{0}{ }^{2} M}
$$

where $M$ - the bending energy of smectic layers.

Under a slow variation of an applied electric field (e.g. the frequency of the triangular shape bipolar voltage $\mathrm{f}<1 \mathrm{~Hz}$ ), the torque of viscous friction practically does not effects on the value of the re-polarization current $I_{P}$. In this case, the dispersion of the susceptibility $\chi_{\text {st }}$ corresponds to the elastic (reversible) process of changing the angle $\psi$ distribution (describing the deformation of smectic layers), when there are weak distortions of a structure and its relaxation to the unperturbed state at decreasing the field strength.

If the reorientation of the dipole moments of molecules occurs in the same phase with the electric field change, that is without a phase shift between the exciting action and the dielectric response of a structure, then the susceptibility $\chi_{\text {st }}$ is a constant (Figure 4).

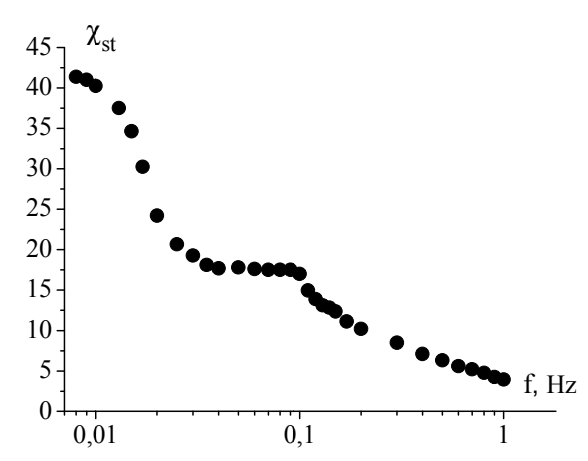

Fig. 4. The frequency dependence of the dielectric susceptibility for FLC with a periodic deformation of smectic layers. The amplitude of triangular shape bipolar voltage is $\pm 0.25 \mathrm{~V}$. The thickness of an electro-optical cell $\mathrm{d}=4 \mu \mathrm{m}$.
In this case, the value of the dielectric susceptibility $\chi_{s t} \approx 17$ corresponds to the reversible process of changing the angle $\psi$ distribution. The energy $M$ of smectic layers deformation, derived from the equation (2), is about $4 \cdot 10^{3} \mathrm{erg} / \mathrm{cm}^{3}$, when the spontaneous polarization $P_{S}=40 \mathrm{nC} / \mathrm{cm}^{2}$ and the tilt angle of molecules in smectic layers $\Theta_{0}=23^{\circ}$.

For FLC with the director helical winding a part of the dependence $\chi_{s t}(f)$, wherein the static susceptibility is independent of the electric field frequency belongs to the range from 1 to $10 \mathrm{~Hz}$ [7].

The bulk density of the free energy associated with this deformation can be written as [3]:

$$
F=\frac{1}{2} K\left(\frac{d \psi}{d y}\right)^{2}+\frac{1}{2} M\left(\frac{l-l_{0}}{l_{0}}\right)^{2}
$$

where $K$ - the elasticity coefficient, which describes the director's deformation over the angle $\psi, M$ - the bending energy of smectic layers. Taking into account the smallness of angles $\psi$ and $\Theta_{0}\left(\psi, \Theta_{0}<<1\right)$ the relative change of the smectic layer thickness $\left(l-l_{0}\right) / l_{0} \cong\left(\psi^{2}-\Theta_{0}^{2}\right) / 2[8]$.

Minimizing the functional (3) we come to the next equation, which describes the distribution of the angle $\psi$ along the coordinate $\boldsymbol{y}(0 \leq y \leq d, d$ is the thickness of the electro-optical test cell):

$$
\frac{d^{2} \psi}{d y^{\prime 2}}+\frac{M \Theta_{0}^{2} d^{2}}{2 K} \psi-\frac{M d^{2}}{2 K} \psi^{3}=0
$$

where $y^{\prime}=y / d, 0 \leq y^{\prime} \leq 1$.

We don't take into account the energy of interaction of FLC molecules with restricting surfaces and propose that

$$
\psi(y=0)=0 \text {, and } d \psi / d y=0 \text { at } \psi=\Theta_{0},
$$

The last condition means that the maximum value of the angle $\psi$ equals to the tilt angle of molecules in smectic layers $\Theta_{0}$. Where the angle $\psi=\Theta_{0}$, the main optical axis (FLC director) deviates by the angle $\Theta_{0}$ relative to the plane of incident light polarization. Spaces with maximum light transmission appear light stripes in Figure 1a. Their striping illustrates periodical changes of the angle $\psi$ along the coordinate $\boldsymbol{y}$.

There are three trivial solutions of the equation (4): $\psi=0$ and $\psi= \pm \Theta_{0}$. The solution $\psi=0$ corresponds to the structure with smectic layers that are orthogonal to the plane of the substrate. Solutions $\psi= \pm \Theta_{0}$ gives the absolute minimum of the free energy $F=0$. Both cases correspond to the structures with inclined smectic layers.

The exact solution of the equation (4) is written in terms of the Jacobi elliptic sine:

$$
\psi=\sqrt{2} \Theta_{0} \frac{k}{\sqrt{1+k^{2}}} \operatorname{sn}\left(\Theta_{0} d \sqrt{\frac{M}{2 K}} \frac{\left(y^{\prime}+C_{1}\right)}{\sqrt{1+k^{2}}}, k\right),
$$

where $k=\sqrt{\frac{M \Theta_{0}^{4} d^{2}}{8 K C_{2}}}-\sqrt{\frac{M \Theta_{0}^{4} d^{2}}{8 K C_{2}}-1}, 0<C_{2}<\frac{M \Theta_{0}^{4} d^{2}}{8 K}$,

i.e. $0<k<1$ is the modulus of Jacobi elliptic sine.

To find the solution of the equation (4) in elementary functions we used the method of Van der Pol [9]. An approximate solution of the equation (4) is the following:

$$
\psi=C_{1} \sin \left(\Theta_{0} \sqrt{\frac{M}{2 K}}\left(1-\frac{3}{8} \frac{C_{1}^{2}}{\Theta_{0}^{2}}\right) y+C_{2}\right)
$$

Substituting boundary conditions (5) into the equation (7) we find constants $C_{1}=\Theta_{0}, C_{2}=0$ and obtain: 


$$
\psi=\Theta_{0} \sin \left(\frac{5}{8} \Theta_{0} \sqrt{\frac{M}{2 K}} y\right)
$$

The analytic solution (7a) describes the structure of smectic layers deformed periodically in the direction, which is orthogonal to substrates (coordinate $y$ ) - Figure 5. Maximums and minimums in the dependence $\psi(y)$ correspond to light and dark stripes in Figure 1.

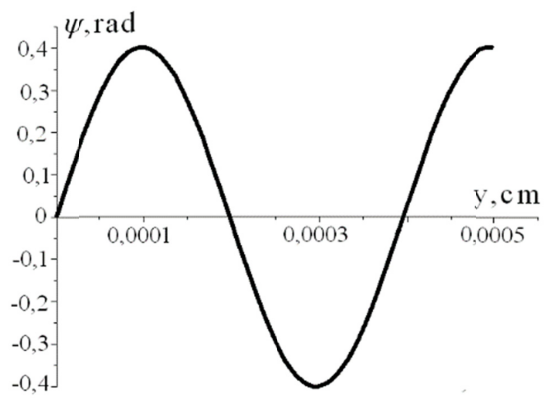

Fig. 5. Graphical presentation of equation (7a) for a cell with FLC layer thickness of $5 \mu \mathrm{m}$. The angle $\Theta_{0}=23^{\circ}, M=4 \cdot 10^{3}$ $\mathrm{erg} / \mathrm{cm}^{3}, K=5 \cdot 10^{-12} \mathrm{~N}$.

Reorientation of FLC director due to the interaction of the alternating electric field $\boldsymbol{E}$ (the field is applied along the coordinate $\boldsymbol{y}$ in Figure 2) with the spontaneous polarization $\boldsymbol{P}_{S}$ can occur by two manners: changing the azimuth angle $\varphi$ of the director orientation by $180^{\circ}$, when the director is reoriented along generating lines of a cone with the apex angle $2 \Theta_{0}$, and changing the angle $\psi$ distribution, describing the deformation of smectic layers. In the first case the dissipative coefficient is the rotational viscosity $\gamma_{\varphi}$ and in the second - the viscosity $\gamma_{\psi}$ of the shear deformation [3].

If the frequency of the external electric field $f \sim 1 / \tau_{m}$, where $\tau_{m}$ - Maxwellian relaxation time (time, during which the shear tensions caused in FLC by the electric field action disappear after cessation of deformation), FLC viscosity is associated with the shear modulus $\mu$ by the following relationship [2]:

$$
\gamma \sim \tau_{m} \cdot \mu
$$

If the time $\tau_{m}$ is independent of the field frequency, FLC viscosity $\gamma_{\varphi}$ does not also depend on the frequency, and the director reorientation time is $\tau_{R} \sim \gamma_{\varphi} / P_{S} \cdot E$ [10]. Accordingly, the viscosity depends on the frequency of the electric field change, when the time $\tau_{m}$ depends on the frequency. In this case, the dissipative factor is the viscosity $\gamma_{\psi}$ of the shear deformation.

The character of FLC director reorientation under the alternating electric field action depends on which of the two dissipative coefficients (rotational or shear viscosity) predominates.

The bulk density of the free energy of the electrostatic interaction can be written as

$$
\frac{1}{2} P_{S} E \cos \varphi_{0}\left(\frac{l-l_{0}}{l_{0}}\right)^{2}
$$

where $\varphi_{0}$ is the initial azimuth angle of the director orientation. Taking into account (9) we minimize the expression for free energy (3) and write the equation of a balance of moments, which describes the change of the angle $\psi$ in the electric field $E$ :

$$
-\gamma_{\psi} \frac{\partial \psi}{\partial t}=K \frac{\partial^{2} \psi}{\partial y^{2}}+\frac{\left(P_{S} E \cos \varphi_{0}+M\right)}{2} \psi\left(\Theta_{0}^{2}-\psi^{2}\right),
$$

where $K \partial^{2} \psi / \partial y^{2}$ and $\gamma_{\psi} \partial \psi / \partial t$ - elastic and viscous moments that define the director reorientation by the angle $\psi$; $K$ and $\gamma_{\psi}$ - the coefficients of elasticity and viscosity; $t$ - time. The electric field switches on at the moment $t=0$.

Equation (10) does not take into account the change of the angle $\Theta_{0}$ in an external field that is correct far from the phase transition to the paraelectric (smectic $A^{*}$ ) phase. Also FLC director reorientation by the azimuth angle $\varphi$ is not considered. Boundary conditions for the equation (10) are similar to the conditions (5) for the equation (4). It is supposed that the distribution (6) remains the same at the start moment.

If to enter variables

$$
\alpha=\frac{d^{2}\left(P_{S} E \cos \varphi_{0}+M\right)}{2 K}, y^{\prime}=\frac{y}{d}, t^{\prime}=\frac{t}{t_{0}}, t_{0}=\frac{\gamma_{\psi} d^{2}}{K},
$$

the equation (10) can be rewritten by such a manner:

$$
\frac{\partial \psi}{\partial t^{\prime}}+\frac{\partial^{2} \psi}{\partial y^{\prime 2}}+\alpha \Theta_{0}^{2} \psi-\alpha \psi^{3}=0 .
$$

Let $\psi$ depends not only on the coordinates but also on the time: $\psi=\psi\left(y^{\prime}, t^{\prime}\right)=\psi_{0} \exp \left(-i \xi t^{\prime}\right)$, where $\xi>0$ - the constant, $\psi_{0}-$ the amplitude, which is a slow function of time [9]. In such a case the equation (10a) transforms to the following:

$$
\frac{\partial \psi_{0}}{\partial t^{\prime}}+\frac{\partial^{2} \psi_{0}}{\partial y^{\prime 2}}+\psi_{0}\left(\alpha \Theta_{0}^{2}+i \xi\right)-\alpha \psi_{0}^{3}=0 \text {. }
$$

Substitution $\psi_{0}=\Phi \exp (-i \eta)$, where $\Phi=\Phi\left(y^{\prime}\right), \eta=\eta\left(t^{\prime}\right)$, to the equation (12) results in a system of two equations:

$$
\left\{\begin{array}{l}
\partial^{2} \Phi / \partial y^{\prime 2}+\Phi \alpha \Theta_{0}^{2}-\alpha \Phi^{3}=0 \\
\Phi\left(\partial \eta / \partial t^{\prime}\right)-\Phi \xi=0
\end{array}\right.
$$

From (13) we have: $\Phi=\frac{\sqrt{2} \Theta_{0} k}{\sqrt{1+k^{2}}} s n\left[\frac{\Theta_{0} \sqrt{\alpha}\left(y^{\prime}+C_{1}\right)}{\sqrt{1+k^{2}}}, k\right]$ and $\eta=\xi t^{\prime}+C_{2}$. Then the function $\psi=\psi_{0} \exp \left(-i \xi t^{\prime}\right)$ can be written as:

$$
\psi=\frac{\sqrt{2} \Theta_{0} k}{\sqrt{1+k^{2}}} \operatorname{sn}\left[\frac{\Theta_{0} \sqrt{\alpha}\left(y^{\prime}+C_{1}\right)}{\sqrt{1+k^{2}}}, k\right] \exp \left(-2 i \xi t^{\prime}-i C_{2}\right)
$$

In the extremely non-linear situation at $k \rightarrow 1$, when the wave period tends to the infinity, we obtain from the relation (14) a spatially localized solution describing the waves of a stationary profile:

$$
\psi=\Theta_{0} t h\left(\frac{\Theta_{0} \sqrt{\alpha}\left(y^{\prime}+C_{1}\right)}{2}\right) \exp \left(-2 i \xi t^{\prime}-i C_{2}\right) .
$$

Taking into account the boundary and initial conditions we find constants $C_{1}=C_{2}=0$ and enter them into (15). Then we obtain:

$$
\psi=\Theta_{0} t h\left(\frac{\Theta_{0} \sqrt{\alpha} y^{\prime}}{2}\right) \exp \left(-2 i \xi t^{\prime}\right) .
$$

Equation (15 a) sets the width of the soliton localization region and its amplitude but does not describe its movement along the coordinate $\boldsymbol{y}$. Transformation

$$
\psi_{0}=\Phi\left(y^{\prime}-V t^{\prime}\right) \exp \left[i(V / 2)\left(y^{\prime}-(V / 2) t^{\prime}\right)\right]
$$

allows to define the soliton velocity $V$ [9]. Then the function $\psi$ will be (16):

$\psi=\sqrt{\frac{2}{\alpha}} \frac{\sqrt{\alpha \Theta^{2}-V^{2} / 4} k}{\sqrt{1+k^{2}}} \operatorname{sn}\left[\frac{\sqrt{\alpha \Theta^{2}-V^{2} / 4} y^{\prime}}{\sqrt{1+k^{2}}}, k\right] \exp \left(i \frac{V}{2}\left(y^{\prime}-V t^{\prime}\right)-i \omega t^{\prime}\right)$,

where $\omega=\xi-V^{2} / 4$ - the frequency in the system of reference moving with a soliton, and $\xi$ - the frequency at the fixed (laboratory) system of reference.

From the relation (16) at $k \rightarrow 1$ we obtain a spatially localized solution describing the wave of a stationary profile moving along the coordinate $\boldsymbol{y}$ with the velocity $V$ : 


$$
\psi=\sqrt{\frac{\omega \Theta^{2}-V^{2} / 4}{\alpha}} t h\left[\sqrt{\frac{\omega \Theta^{2}-V^{2} / 4}{2}}\left(y^{\prime}-V t^{\prime}\right)\right] \exp \left(i \frac{V}{2}\left(y^{\prime}-V t^{\prime}\right)-i \omega t^{\prime}\right)
$$

Consequently, spatially localized solution of the equation (10) is a two-parametric soliton: one of its parameters is the velocity $V$ of moving the soliton center, and the other one - its eigenfrequency $\omega$ in the system of reference moving with the soliton. The solution (17) in graphical form is presented in Figure 6a.

a).

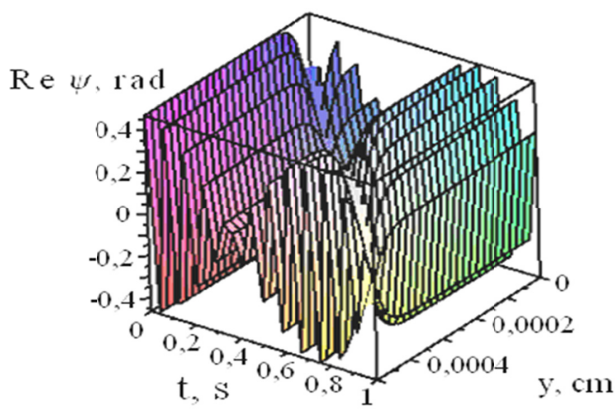

b).

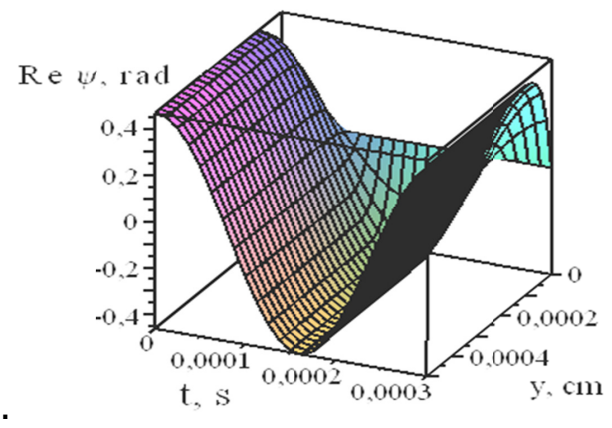

Fig. 6. Graphical presentation of the solution (17), which describes the change of the angle $\psi$ in the electric field $E$ (a) and motion of the orientation bend in FLC layer (b). $E=3 \mathrm{~V} / \mu \mathrm{m}$, $P_{S}=50 \mathrm{nC} / \mathrm{cm}^{2}, M=4 \cdot 10^{3} \mathrm{erg} / \mathrm{cm}^{3}, K=5 \cdot 10^{-12} \mathrm{~N}, \gamma_{\psi}=0.2$ Poise, $\Theta_{0}=23^{\circ}, \varphi_{0}=30^{\circ}$.

Thus, the transition to Maxwellian mechanism of the energy dissipation (dissipative coefficient is a shear viscosity $\gamma_{\psi}$ ) results in the appearance of a soliton, which is a wave packet with localized therein the periodic wave.

Due to the normalization (11) the maximum speed of soliton motion can be written as:

$$
V=2 \Theta_{0} \sqrt{\alpha} K /\left(\gamma_{\psi} d\right)=\left(\Theta_{0} / \gamma_{\psi}\right) \sqrt{2 K\left(M+P_{S} E \cos \varphi_{0}\right)} .
$$

Let $\omega=0$, that is the frequency $\xi=V^{2} / 4$. Then taking into account the normalization (11) the characteristic time of director reorientation due to the movement of the orientation bend (Fig. $6 \mathrm{~b})$, is as following:

$$
\tau_{C}=\frac{\gamma_{\psi} d^{2}}{K \xi}=\frac{2 \gamma_{\psi}}{\Theta_{0}^{2}\left(P_{S} E \cos \varphi_{0}+M\right)} .
$$

If $\varphi_{0}=30^{\circ}, P_{S}=50 \mathrm{nC} / \mathrm{cm}^{2}, M=4 \cdot 10^{3} \mathrm{erg} / \mathrm{cm}^{3}, K=5 \cdot 10^{-12} \mathrm{~N}, E=3$ $\mathrm{V} / \mu \mathrm{m}, \Theta_{0}=23^{\circ}$ и $\gamma_{\psi}=0.2$ Poise, then the speed of soliton center movement $\mathrm{V}=0,65 \mathrm{~cm} / \mathrm{s}$, and reorientation time of the director $\tau_{C} \approx 150 \mu \mathrm{s}$.

The necessary condition for the light scattering is a violation of the optical homogeneity of a medium when its refractive index is not constant and varies throughout all FLC volume. Light scattering in mentioned above helix-free FLC is due to the spatial inhomogeneity of the optical anisotropy.

Scattering occurs on boundaries of spontaneously ordered regions, which are formed in FLC volume at the appearance of nonlinear spatially localized waves of stationary profile (solitons). This results in the formation of transient domains (scattering centers), when inhomogeneous distribution of the electric polarization appears along the smectic layers.

Scattering occurs again after changing the sign of the electric field and disappears, when the motion of solitons reorients the director in all smectic layers, that is, the formation of a homogeneous structure comes to the end, and transient domains disappear. Changing the direction of the electric field induces transient domain formation again, and the process repeats.

\section{EXPERIMENTAL RESULTS AND THEIR DISCUSSION}

As shown above, if a dissipative factor - rotational viscosity $\gamma_{\varphi}$ then reorientation of the director takes place simultaneously in all smectic layers (volume mode of switching), and a time of reorientation $\tau_{R} \sim \gamma_{\varphi} / P_{S} E$ does not depend on the frequency of the electric field change [9].

As a consequence, in our case the electro-optical response time $\tau_{0.1-0.9}$ does not depend on the electric field frequency, if the frequency does not exceed $200 \mathrm{~Hz}$ (Figure 7, curve 1).

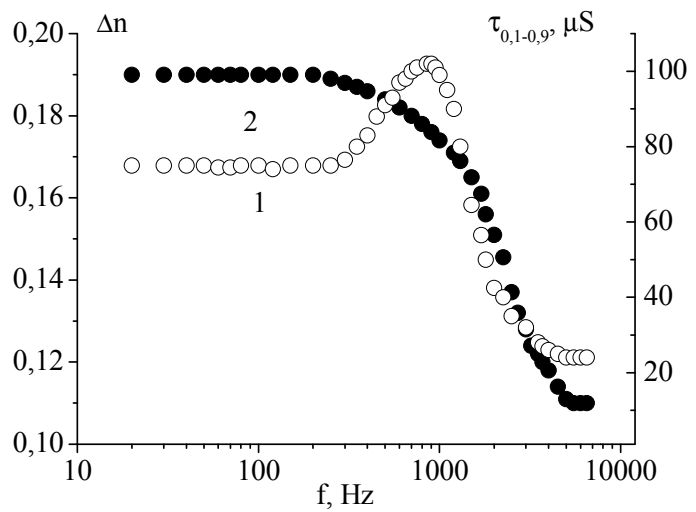

Fig. 7. The frequency dependences of the optical response time (curve 1) and the index of birefringence (curve 2). The thickness of FLC cell $d=13 \mu \mathrm{m}$. The amplitude of bipolar control voltage of rectangular shape (meander) is $\pm 35 \mathrm{~V}$.

In this frequency range the time of relaxation to undisturbed state after the electric field switching off also does not depend on the frequency of electric field change (Figure 8).

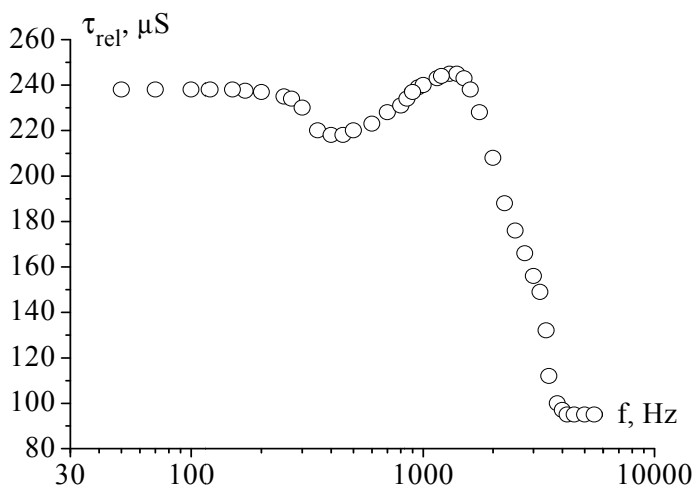

Fig. 8. The frequency dependence of the FLC director's relaxation time. The electro-optical cell thickness is $13 \mu \mathrm{m}$. The amplitude of control voltage (rectangular pulses) is $35 \mathrm{~V}$.

The relaxation time was measured as the time during of which the surface charge on the cell electrodes (it is proportional to the integral of repolarization current $I_{P}$ ) reduces to zero. Rectangular (unipolar or bipolar) pulses were 
supplied to electrodes of the electro-optical cell, and the pulse duration was equal to the interval between pulses.

Transition to the Maxwellian relaxation time occurs, when the time of the director relaxation begins to depend on the frequency of the electric field change (Figure 8).

According to (8), if the relaxation time depends on the electric field frequency, a dissipative coefficient, that is the shear viscosity $\gamma_{\psi}$, also must depend on the frequency.

The transition to Maxwellian mechanism of energy dissipation is accompanied with strong frequency dependence of the optical response time $\tau_{0.1-0.9}$ (Figure 7, curve 1). The initial increase of the time $\tau 0.1-0.9$ is due to the simultaneous presence of two dissipative coefficients $\gamma_{\varphi}$ and $\gamma_{\psi}$. Moreover time $\tau_{0.1-0.9}$ increases no more than 25\%, that's allows to suppose the magnitude of the shear viscosity $\gamma_{\psi}$ in this case does not exceed 0.2 poise (the magnitude of the rotational viscosity of used FLC $\gamma_{\varphi}=0,7$ Poise).

In turn, the predominance of the shear viscosity results in a reduction of time $\tau_{0.1-0.9}$ in several times. A further increase in frequency, when the transition to the soliton mode takes place, and time $\tau 0.1-0.9$ is determined by a velocity of soliton wave motion, the frequency dependence of an optical response is practically absent (Figure 7, curve 1).

The transition to Maxwellian mechanism of dissipation energy is also accompanied with decreasing the index of FLC birefringence $\Delta \mathrm{n}$ (Figure 7, curve 2). The maximum change $\Delta \mathrm{n}$ (1.5 times) takes place at the transition to the soliton mode.

The light scattering efficiency is usually characterized by a contrast ratio, i.e. the ratio of the intensity of the light spreading in a straight direction without scattering and the intensity of light scattering into a sufficiently small solid angle, which was formed in our case by a diaphragm of $1.5 \mathrm{~mm}$ diameter located behind an electro-optical cell.

When operating in light scattering mode the polarizers are not used, and the light transmission of electro-optical cell reaches up to $80 \%$. It was mainly limited by the transparent conductive coatings deposited on glass substrates.

The light scattering efficiency and light transmittance of an electro-optical cell are defined both by a frequency and amplitude of the control voltage. For fixed electric field strength (or the amplitude of bipolar pulses of the control voltage) the maximal efficiency of light scattering (contrast ratio) and maximal light transmission of an electro-optical cell are achieved at a different duration of pulses (Figure 9).

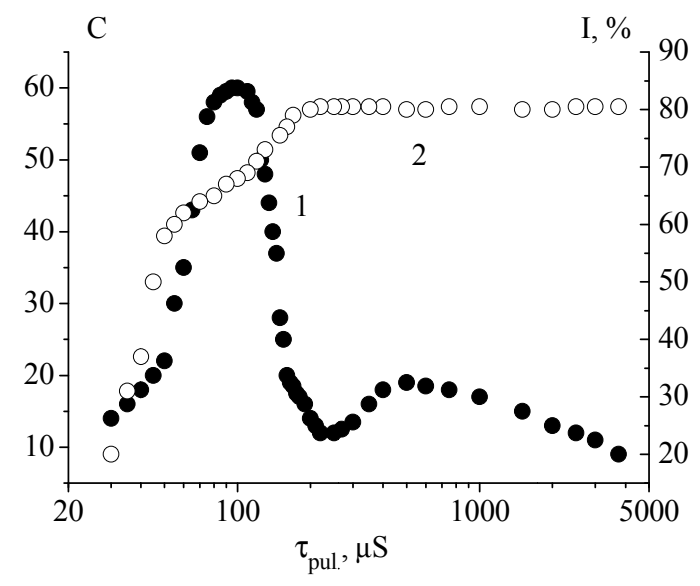

Fig. 9. The dependences of the light scattering efficiency $C$ (curve 1) and light transmission $I$ (curve 2) on the duration of bipolar control voltage pulses at fixed amplitude. The pulse amplitude is $\pm 35 \mathrm{~V}$. The thickness of FLC cell is $13 \mu \mathrm{m}$.
This means the time of the formation of a regular structure of transient domains and time of the complete disappearance of this structure are distinguish. So, the pulse duration required to switch on a state with maximum light transmission, which corresponds to complete absence of transient domains, is $1.5 \div 2$ times longer (Figure 9, curve 2). If the pulse duration is less than the minimal time required for complete disappearance of transient domains, the light transmittance of a cell decreases.

Maximum light scattering efficiency is achieved, when the regular structure of scattering centers arises in the form of circular domains distributed enough evenly over FLC volume.

Reducing the thickness of an electro-optical cell shifts the scattering peak corresponding to the regular scattering structure to shorter pulse durations, but the contrast ratio is reduced also.

At the inversion of an electric field sign (i.e. change of the pulse polarity) insufficient duration of the field action (small pulse duration) does not allow to form a regular structure of transient domains that results in a decrease of the scattering centers density, and as a result, the light scattering efficiency is decrease.

On the other hand, for fixed pulse duration to obtain a maximum light transmission of a cell the higher amplitude of pulses is required than for achieving the maximum efficiency of light scattering.

When a certain relations between the amplitude and duration of the control voltage bipolar pulses, the energy of deformation of smectic layers and FLC spontaneous polarization, the period of layers deformation and electrooptical cell thickness, are reached the scattering process shows the bistable character.

This means that both optical states of the structure (with maximum transmittance and maximum light scattering efficiency) are stored for several tens of seconds after an electric field switching off or till the arrival of pulse opposite polarity (Figure 10).

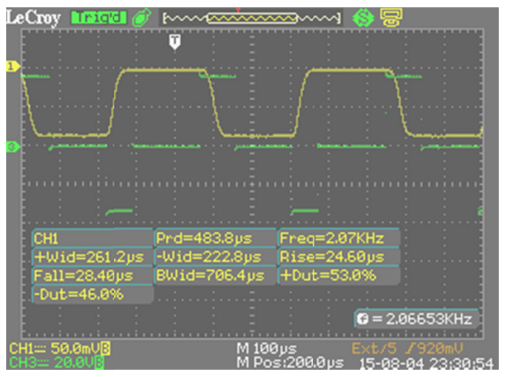

Fig. 10. Oscillogramms of the control voltage (green light channel CH3) and optical response (yellow light - channel CH1) for a bistable switching mode. Control voltage: bipolar pulses of the amplitude $\pm 35 \mathrm{~V}$ (zero level is an arrow with digit 3 ). The frequency of light modulation is $2 \mathrm{kHz}$. The upper level of an optical response is a scattering state, the bottom level - a transparent state (zero level of light transmission is an arrow with digit 1). The thickness of an electro- optical cell is $13 \mu \mathrm{m}$. Optical response time on the rising edge - Rise, on the falling edge - Fall.

Switching by voltage pulses of different duration provides the operation of an electro-optical cell in the bistable mode with a light transmission of about $80 \%$ and a contrast ratio of above 200:1.

There is an optimal ratio between the period of deformation of FLC smectic layers and a thickness of electro-optical cell, when at a certain strength of the electric field the velocity of the soliton waves motion is maximum (respectively, the electro- 
optical response time $\tau_{0.1-0.9}$ is minimum), and the light modulation frequency is also maximum.

For used FLC with a period of layers deformation of the order of $2 \mu \mathrm{m}$ the optimal thickness of an electro-optical cell is of about $20 \mu \mathrm{m}$. So, for the cell thickness $\mathrm{d}=18 \mu \mathrm{m}$ the maximal light modulation frequency is about $5 \mathrm{kHz}$ (the time $\tau_{0.1-0.9}$ does not exceed $12 \mu \mathrm{s}$ ) at the field strength of $2.7 \mathrm{~V} / \mu \mathrm{m}$ (Figure $11 \mathrm{a}$ ). At the same field strength for the cell thickness of $13 \mu \mathrm{m}$ the maximal light modulation frequency does not exceed $2 \mathrm{kHz}$, and the time $\tau_{0.1-0.9}$ is of $30 \mu$ s (Figure 10).

Changing the on-off time ratio between control voltage pulses (while maintaining a duration) results in a change of the ratio between the time of existence of both optical states (Fig. $11 \mathrm{~b})$.

a
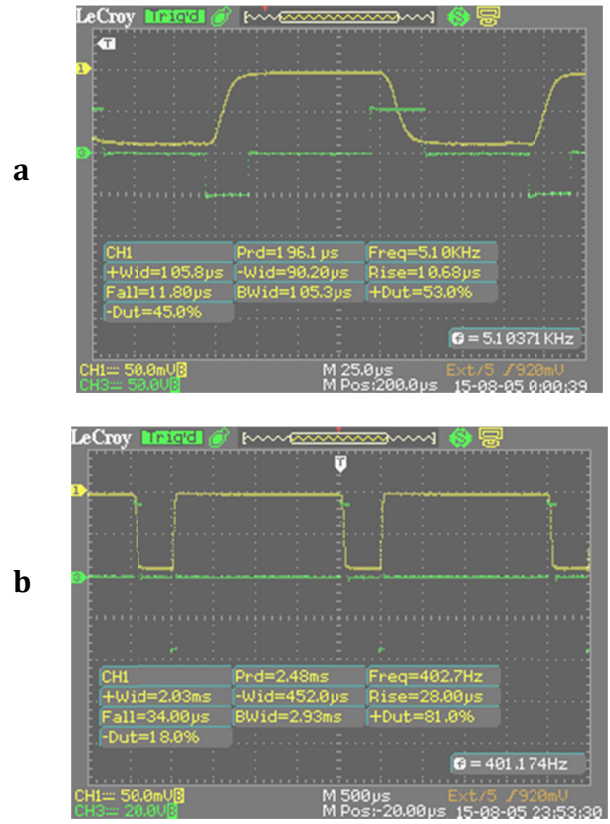

Fig. 11. Oscillogramms of the control voltage (green light channel CH3) and optical response (yellow light - channel CH1): a - at the amplitude of voltage pulses $\pm 48 \mathrm{~V}$ and light modulation frequency of $5 \mathrm{kHz}$; $\mathrm{b}$ - at changing the on-off time ratio between voltage pulses of the amplitude $\pm 35 \mathrm{~V}$ at the light modulation frequency is of $400 \mathrm{~Hz}$. The thickness of an electrooptical cell is $18 \mu \mathrm{m}$. The upper level of an optical response is a scattering state, the bottom level - a transparent state (zero level of light transmission is an arrow with digit 1). Optical response time on the rising edge - Rise, on the falling edge Fall.

The minimum lifetime of states with the highest light transmission and maximum efficiency of light scattering cannot be less than the sum duration of bipolar voltage pulses, which switch on and switch off the scattering process.

Times ON and OFF of the scattering process (electro-optical response time $\tau_{0.1-0.9}$ ) are almost the same and do not exceed the minimum duration of a pulse, which switches on the scattering process (Figure 10).

Dependence $\tau_{0.1-0.9}(E)$ measured at the control voltage frequency $\mathrm{f}=1 \mathrm{kHz}$ (Figure 12, curve 1 ) corresponds to the case of influence of both dissipative coefficients $-\gamma_{\varphi}$ and $\gamma_{\psi}$ (Figure 7 , curve 1).

The prevalence of the shear viscosity $\gamma_{\psi}$ (the frequency of an electric field change more than $1 \mathrm{kHz}$ ) results in reducing T0.1-0.9 in several times (Figure 12, curve 2).

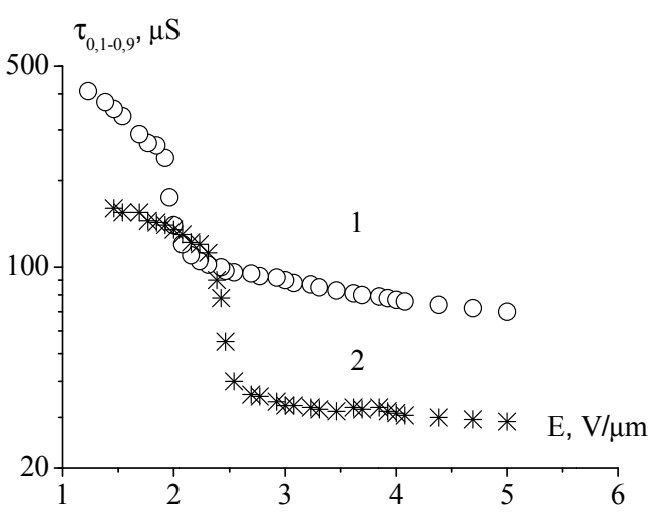

Fig. 12. The field dependence of the optical response time (time $\mathrm{ON}$ and OFF of the scattering process) for FLC electro-optical cell with the thickness of $13 \mu \mathrm{m}$. The control voltage frequency is $1 \mathrm{kHz}$ (curve 1 ) and $3 \mathrm{kHz}$ (curve 2).

The transition to the soliton mode, both at low and high frequencies, is accompanied with a sharp decrease of the electro-optical response time, when a certain threshold of electric field strength is achieved. In the soliton mode the response time $\tau_{0.1-0.9}$ is almost independent of the field frequency change. With increasing the electric field frequency the threshold value of the electric field strength increases for the transition to the soliton mode (Figure 12).

A few maximums of light scattering efficiency can be observed in the dependence on the duration of the electric field action (duration of control voltage pulses) and electro-optical cell thickness (Figure 13). The appearance of the second and third maximums of light scattering efficiency occurs when the thickness of an electro-optical cell increases (compare Figures 9 and 13).

Increasing the pulse duration results in increasing the length of domain walls and leads to irregular scattering structures. As a consequence, the density of scattering centers reduces, that is a reason light scattering efficiency decreasing (Figure 13).

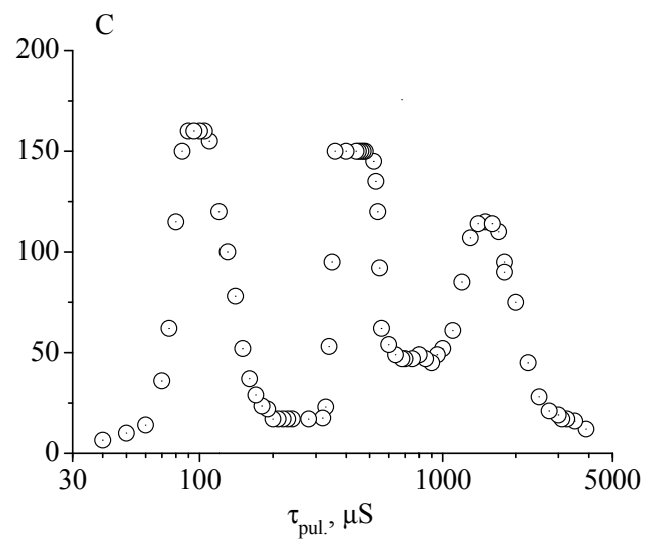

Fig. 13. The dependence of the light scattering efficiency on the duration of control voltage pulses at a fixed their amplitude. The pulse amplitude is of $\pm 50 \mathrm{~V}$. The thickness of an electrooptical cell is $18 \mu \mathrm{m}$.

The transitions between light-scattering modes (correspond to maximums of the light scattering efficiency) result in the chaotic change in the position of the scattering indicatrix, while the duration of voltage pulses supplied simultaneously to an electro-optical cell corresponds to different maximums. Due to short-term light scattering 
switching on (less than for $50 \mu \mathrm{s}$ ) the structures are formed in FLC with almost random distribution of refractive index gradients over all FLC layer, which is the reason of a spatially non-uniform (across the beam) phase modulation of light in an electro-optical cell.

Spatially non-uniform modulation of the phase delay, the depth of which is the order and more $\pi$, allows to destroy the phase relations in a laser beam passing through an electrooptical cell and, consequently, to suppress the speckle noise in images formed by a laser beam [11].

The scattering diagram is asymmetric relative to a plane, which is perpendicular to the direction of the incident light (Figure 14). The efficiency of light scattering is maximum in the direction coinciding with the direction of the incident light $\left(\Phi=0^{\circ}\right)$, and decreases in the opposite direction $\left(\Phi=180^{\circ}\right)$.

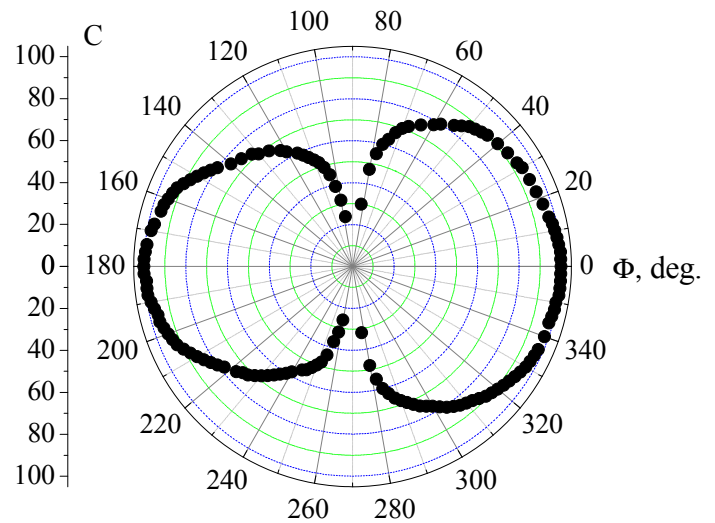

Fig. 14. The dependence of the light scattering efficiency on the viewing angle (direction of scattering) along the smectic layers. The thickness of an electro-optical cell is $13 \mu \mathrm{m}$. Bistable mode of switching. Control voltage: bipolar pulses with the amplitude of $\pm 35 \mathrm{~V}$.

Reduction of light scattering efficiency in the directions located at angles than more $20^{\circ}$ relative to the direction of the incident light (and to the opposite direction) is due to the irregular distribution of the density of scattering centers along smectic layers (along the coordinate $y$ in Figure 2). This leads to a change in conditions of interference: light emission of scattering centers interferes at the unequal phase difference.

\section{CONCLUSION}

A new type of helix-free FLC is considered, where reorientation of the director (main optical axes) is due to the movement of spatially localized waves with a stationary profile - solitons arising at the transition to the Maxwellian mechanism of energy dissipation. The spatially-periodical deformations of the smectic layers observed experimentally earlier are described theoretically now.

The process of intensive light scattering on a dynamic domain structure in such FLC takes place, and this process has a bistable character at a certain ratio between the amplitude and duration of the control voltage bipolar pulses, the energy of the smectic layers deformation and FLC spontaneous polarization, the period of layer deformation and electrooptical cell thickness.

This means that both optical states of the structure - with a maximum transmittance and the maximum light scattering efficiency - can be memorized for several tens of seconds after switching off an electric field or till the arrival of a pulse of opposite polarity. Switching by voltage pulses of different duration provides the operation of an electro-optical cell in the bistable mode with the light transmission of $80 \%$, contrast ratio over 200:1 and response time of a few tens of microseconds. These unique properties are due to FLC composition and selected control regime.

Changing the channel of energy dissipation allows to reduce the electric field strength (at which the maximum efficiency of light scattering is achieved) down to $2 \div 3 \mathrm{~V} / \mu \mathrm{m}$ and to increase the frequency of light modulation up to $3 \div 5 \mathrm{kHz}$. In addition, a strong dependence of the light-scattering efficiency on the amplitude, pulse recurrence frequency and pulse duration of the control voltage allows controlling the scattering process (changing the light transmission of an electro-optical cell) by means of changing both the duration and the amplitude of control voltage pulses.

Due to short-term (less than $50 \mu \mathrm{s}$ ) switching on light scattering the structures are formed in FLC with almost random distribution of refractive index gradients over all FLC layer, which in turn are the causes of a spatially non-uniform (across the beam) phase modulation of light in an electro-optical cell. This allows to destroy the phase relations in a laser beam passing through an electro-optical cell and, consequently, to suppress effectively the speckle noise in images formed by a laser beam.

At last, the phenomena of intensive and fast light scattering can be effectively used in polarizer-free light modulators including IR and spatial ones.

Funding. The Russian Foundation for Basic Research (RFBR) (14-0700185-a, 14-07-92601-a); The Program of Basic Research of the Presidium of the Russian Academy of Sciences.

Acknowledgment. The authors thank A.V. Novozhenov for the assistance in this work.

\section{References}

1. A.L. Andreev, T.B. Andreeva, I.N. Kompanets, Y.P. Bobylev, V.M. Shoshin, M.V. Minchenko. Electrically Controlled Light Scattering in Helix Ferroelectric Liquid Crystals. J. of Optical Technology, 77, No.12, 776-783 (2010).

2. T. Fedosenkova, A. Andreev, E. Pozhidaev, I. Kompanets. Electrically birefringence controlled by external electric field in helix-free ferroelectric liquid crystals. Bulletin of the Lebedev Physics Institute, No. 3, 36-42 (2002).

3. L.D. Landau, and E.M. Lifshits. Theory of Elasticity. Publ. House "Nauka", Moscow, 188-189 (1987).

4. A.L. Andreev, T.B. Andreeva, I.N. Kompanets, N.V. Zalyapin. Optical response of helix-free FLC: continuous gray scale, fastest response, and lowest control voltage. Journal of the SID, v. 22, Issue 2, 115-121 (2014).

5. S.A. Pikin. Structural transformations in liquid crystals. Publishing House "Nauka", Moscow, 167-169 (1981).

6. B. Urbanc, B Zeks, T. Carlsson. Nonlinear effects in the dielectric response of ferroelectric liquid crystals. Ferroelectrics, v. 113, 219230 (1991).

7. A.L. Andreev, E.P. Pozhidaev, I.N. Kompanets, T.B. Fedosenkova, V.Ya. Zyryanov, S.L. Smorgon, T. Weyrauch, W. Haase. Saturation voltage and elastic energy of polymer dispersed ferroelectric liquid crystal films. Ferroelectrics, v. 243, 189-196 (2000).

8. J. Pavel, M. Glogarova. A new type of layer structure defects in chiral smectics. Liquid crystals, v. 9, No.1, 87-93 (1991).

9. A.M. Kosevich, A.S. Kovalev. Introduction to Nonlinear Physical Mechanics. Publ. House "Naukova Dumka", Kiev (1988).

10. M.A. Handschy, N.A. Clark and S.T. Lagerwall. Field-Induced FirstOrder Orientation Transitions in Ferroelectric Liquid Crystals. Phys. Rev. Lett, 51, 471-474 (1983).

11. A.L. Andreev, T.B. Andreeva, I.N. Kompanets, N.V. Zalyapin. Speckle noise suppression using a helix-free ferroelectric liquid crystal cell. Quantum Electronics, 44, No. 12, 1136 - 1140 (2014). 